\title{
Temporal cues derived from statistical patterns can overcome resource limitations in the attentional blink
}

\author{
Troy A. W. Visser • Jeneva L. Ohan • James T. Enns
}

Published online: 27 March 2015

(C) The Psychonomic Society, Inc. 2015

\begin{abstract}
Previous studies have shown that humans are sensitive to statistical patterns indicating the likely locations, identities, and timings of visual targets. Here we tested whether participants can also use this kind of information to ameliorate the attentional blink $(\mathrm{AB})$ - a reduction in accuracy for the second of two targets $(\mathrm{T} 1, \mathrm{~T} 2)$ presented at brief intertarget intervals (lags). In particular, we asked whether participants can use patterns arising from differential distributions of intertarget lags across trials to predict the arrival of $\mathrm{T} 2$. We tested this by comparing the $\mathrm{ABs}$ in an aging versus a nonaging distribution of trials, where aging refers to the increased likelihood of $\mathrm{T} 2$, given that it has not yet occurred, when lags occur with equal frequencies. Experiments 1 and 2 showed that the aging condition yielded greater $\mathrm{T} 2$ accuracy at longer lags than did the nonaging condition. In Experiment 3, we used a more sensitive response time measure to show faster $\mathrm{T} 2$ discrimination at shorter lags in the nonaging condition. These results demonstrate that participants can predict the likely onset of $\mathrm{T} 2$ by using statistical patterns present in the AB task, and that they can use this ability to more effectively direct limited processing resources.
\end{abstract}

Keywords Divided attention $\cdot$ Attentional blink $\cdot$ Precuing

Our ability to predict future events benefits many tasks. For example, visual search efficiency is increased when

T. A. W. Visser $(\bowtie) \cdot J$. L. Ohan · J. T. Enns

School of Psychology (M304), University of Western Australia,

35 Stirling Hwy, Crawley, Western Australia 6009, Australia

e-mail: troy.visser@uwa.edu.au

\section{J. T. Enns}

Department of Psychology, University of British Columbia

2136 West Mall, Vancouver V6T 1Z4, British Columbia, Canada the locations of key elements in a display are repeated across trials (Chun \& Jiang, 1998) and when target objects are semantically congruent with their surroundings (Biederman, Mezzanotte, \& Rabinowitz, 1982). Similarly, validly cueing the location of a future target significantly enhances both perceptual and response-related target processing, whereas the opposite is true for targets presented at unexpected locations (Henderson, 1991; Nakayama \& Mackeben, 1989; Posner, 1980; Posner, Snyder, \& Davidson, 1980; Yeshurun \& Carrasco, 1999). These same patterns of performance carry over to real-world scenarios, such as motor vehicle operation (Engström, Aust, \& Vistrom, 2010; Ho, Tan, \& Spence, 2005).

In addition to knowing where a target may appear, and what it might be, knowing when a target will appear is also of great value. For example, a seminal study by Coull and Nobre (1998) found that cues that accurately predicted the onset of a target benefited detection in a similar way to a cue indicating the target's spatial location. Correa, Lupiáñez, and Tudela (2005) observed increased discrimination sensitivity for targets presented at a cued temporal interval relative to targets presented at unexpected times. Finally, validly cueing a target's onset can allow it to escape the negative consequences of inattention (e.g., Hilkenmeier \& Scharlau, 2010; Martens \& Johnson, 2005; Shen \& Alain, 2011; Tang, Badcock, \& Visser, 2014; Visser, Tang, Badcock, \& Enns, 2014).

Although these studies have provided evidence that temporal cues can help participants overcome resource limitations, it is still unclear whether participants can generate predictions about when targets will appear on the basis of temporal regularities present in the task environment. Much of the previous research in this area has been done in the context of the rapid serial visual presentation (RSVP; e.g., Broadbent \& Broadbent, 1987; Potter, 1976; Raymond, Shapiro, \& Arnell, 1992) paradigm. Here, stimuli are presented rapidly 
$(\sim 10 \mathrm{~Hz})$ at a central, fixated location and participants are instructed to detect or identify one or more targets (for variations, see Visser, Zuvic, Bischof, \& Di Lollo, 1999; Ward, Duncan, \& Shapiro, 1997). When the RSVP stream contains two targets, performance on the first (T1) is typically quite good, whereas performance on the second (T2) is poor at intertarget intervals (lags) of less than about $500 \mathrm{~ms}$. This so-called attentional blink ( $\mathrm{AB}$ ) arises from an inability to adequately allocate processing resources to $\mathrm{T} 2$ as a result of the need to process T1 (e.g., Chun \& Potter, 1995; Di Lollo, Kawahara, Ghorashi, \& Enns, 2005; Olivers \& Meeter, 2008; Raymond et al., 1992; Taatgen, Juvina, Schipper, Borst, \& Martens, 2009; Wyble, Bowman, \& Nieuwenstein, 2009; see Dux \& Marois, 2009, and Martens \& Wyble, 2010, for reviews).

Several studies have shown that conveying explicit information about the expected onset of $\mathrm{T} 2$ can reduce the $\mathrm{AB}$ deficit. For example, Hilkenmeier and Scharlau (2010) found that $\mathrm{T} 2$ accuracy increased when $\mathrm{T} 1$ identity signaled the most likely T1-T2 lag, whereas Martens and Johnson (2005; see also Visser et al., 2014) found similar results using a cue presented prior to each trial. Shen and Alain (2011) showed that instructing participants at the beginning of a block of trials to focus attention at a specific time interval following $\mathrm{T} 1$ strongly benefited $\mathrm{T} 2$ accuracy when it appeared at that interval. Finally, both Choi and colleagues (Choi, Chang, Shibata, Sasaki, \& Watanabe, 2012; Choi \& Watanabe, 2014) and Tang et al. (2014) showed a reduction in the AB following a training regimen consisting of several hundred trials presented at a constant intertarget lag with a uniquely colored $\mathrm{T} 2$, whereas Nieuwenstein, Chun, van der Lubbe, and Hooge (2005) found that identification of a unique, red T2 was facilitated by the presentation of an earlier red item in the RSVP stream.

To date, however, the evidence that participants can derive processing benefits from temporally predictable events in the $\mathrm{AB}$ task has been equivocal. On the one hand, Badcock, Badcock, Fletcher, and Hogben (2013) found that the AB was ameliorated when $\mathrm{T} 1$ was preceded by a distractor stream of a predictable duration. This implies that being able to reliably predict $\mathrm{T} 1$ onset aids $\mathrm{T} 2$ processing. On the other hand, Martens and Johnson (2005) failed to find benefits of presenting a series of trials at a constant intertarget lag, and Visser et al. (2014) obtained a benefit under these conditions only when participants were explicitly alerted to its presence. This implies that a reliably predictive task environment is, by itself, insufficient to allow participants to make predictions about when T2 will be presented. Consistent with this conjecture, in their second experiment, Hilkenmeier and Scharlau (2010) found greater temporal cuing benefits when participants were informed that $\mathrm{T} 1$ identity predicted $\mathrm{T} 1-\mathrm{T} 2$ lag than when participants were unaware of this contingency. The dependence of these effects on explicit instructions is surprising when considered with other evidence that humans are highly sensitive to statistical patterns and regularities, and that they are able to use this information without instruction in many tasks to selectively process task-relevant features (TurkBrowne, Scholl, Chun, \& Johnson, 2009; Zhao, Al-Aidroos, \& Turk-Browne, 2013).

To investigate this issue, we capitalized on a phenomenon known as the foreperiod effect (Gabay \& Henik, 2008, 2010; Niemi \& Näätänen, 1981), in which observers respond more quickly and accurately to a target stimulus when it is preceded by a warning signal that can be used to predict target onset. This improvement appears to arise at multiple levels, including the motor system (Burle, Tandonnet, \& Hasbroucq, 2010; Sanders, 1998), response selection mechanisms (Hackley, 2009; Los \& Schut, 2008), and perception (Bausenhart, Rolke, \& Ulrich, 2008; Correa et al., 2005; Rolke \& Hofmann, 2007). A foreperiod arises in most conventional $\mathrm{AB}$ experiments because all trials contain two targets that are presented with equal frequency at each potential lag. As a result, probabilistic information about the occurrence of $\mathrm{T} 2$ is contained in the time that has elapsed since the onset of T1. That is, presentation of $\mathrm{T} 1$ serves as a warning that $\mathrm{T} 2$ is upcoming, with the likelihood of T2 appearing increasing directly with the passage of time since $\mathrm{T} 1$. To illustrate, if there are 25 trials at each of four lags, the probability of the target appearing at the first lag is .25 (25/100); however, given that it does not appear at the first lag, the probability that the target will appear in the second lag increases to $.33(25 / 100-25)$, the probability at the third lag increases to $.50[25 /(100-25-$ $25)]$, and if the target has not appeared by the third lag, the probability that it will appear at the longest lag is $1[25 /(100-$ $25-25-25)]$. This is referred to as an "aging" distribution with regard to lag (Näätänen, 1970).

In Experiment 1, we tested whether participants are sensitive to this temporal cue for $\mathrm{T} 2$ by comparing performance on an "aging" distribution of trials (all lags equally frequent) which is the distribution of lags across trials conventionally used in the $\mathrm{AB}$ task - with performance on a "nonaging" distribution of trials (Näätänen, 1970) in which the number of trials at each lag decreased by half with each step in lag. With this modification, the probability of $\mathrm{T} 2$ onset remains constant as the time elapsed since $\mathrm{T} 1$ increases. Thus, if participants are sensitive to the temporal information given by the aging distribution then the $\mathrm{AB}$ should be larger (i.e., T2 accuracy should be poorer) in the nonaging condition relative to the aging condition, with this difference being greatest at the longest lag, where the greatest disparity in temporal information is provided by the passage of time from $\mathrm{T} 1$ onset.

Although this manipulation of trial frequency across lags allowed us to vary the temporal information provided by the passage of time following $\mathrm{T} 1$, it also introduced a second possible source of temporal information, by altering the a priori probability of trials occurring at a given lag. If participants are sensitive to this information, they should be able to 
predict the likely lag that $\mathrm{T} 2$ will be presented at on each trial, in a manner similar to that of the participants in Martens and Johnson (2005) and Visser et al. (2014), who received every trial at the same lag. Thus, we would expect that the $\mathrm{AB}$ should be smaller in the nonaging than in the aging condition at the shortest lag, whereas the opposite pattern should be found at the longest lag, reflecting the fact that these lags have the largest disparity in a priori probabilities between conditions.

\section{Experiment 1}

\section{Method}

Participants Twenty undergraduate students (eight female, 12 male; mean age 20.15 years) were recruited in exchange for partial credit toward course completion. All provided informed consent prior to participating, had normal or corrected-to-normal vision and were naïve about the purpose of the experiment.

Apparatus and stimuli The stimuli were presented on a 19-in. (viewing size: 18 in.) Dell M992 monitor running at a refresh rate of $100 \mathrm{~Hz}$, attached to a Pentium computer running Presentation software (Version 16.20; Neurobehavioral Systems), located in a dimly lit room. The software also recorded responses from the computer keyboard.

All stimuli subtended a visual angle of approximately $1^{\circ}$ at a viewing distance of $60 \mathrm{~cm}$. Targets were dark gray (RGB: $70,70,70)$ and consisted of all letters of the English alphabet except I, O, Q, and Z, which were omitted due to their structural similarity to the digits $1,0,2$, and 7 . Distractors were light gray (RGB: 250, 250,250) and consisted of the digits 19. Targets and distractors were presented in 28-point Arial font.

Procedure Participants completed two blocks of trials in counterbalanced order. In the "aging" condition, a conventional $\mathrm{AB}$ paradigm was presented, with 60 trials at each of four lags. In the "nonaging" condition, the proportion of trials decreased as lag increased, such that 128 trials were presented at lag 2,64 trials at lag 3,32 trials at lag 5, and 16 trials at lag 7. In both conditions, 16 "catch" trials were also included, in which the second target was omitted and replaced with a distractor digit. This yielded a total of 256 trials in both conditions. Given this distribution of trials, in the aging condition, the probability that $\mathrm{T} 2$ would be presented (given that it had not already appeared) at lag 2 was .23, at lag 2 was .31, at lag 5 was .44, and at lag 7 was .79; in the nonaging condition, the probability that $\mathrm{T} 2$ would be presented (given that it had not already appeared) was .50 across all lags. Participants were informed that some trials would contain only one target and to respond appropriately, but they were not told about variations in the numbers of trials per lag.

Each trial began with a fixation cross at the center of the screen. When participants were fixated on this cross, they pressed the space bar to initiate an RSVP stream. Each item in the stream was presented for $30 \mathrm{~ms}$ and separated by a 60 ms blank interstimulus interval. The RSVP began with a randomly determined five to eight distractor digits. The digits were chosen randomly, with the proviso that the same digit could not appear consecutively. Next, the first target letter was presented, followed by one, two, four, or six additional distractor digits, and then the second target letter. This yielded intertarget stimulus onset asynchronies of $180,270,450$, and $630 \mathrm{~ms}$ (lags 2, 3, 5, and 7, respectively). Targets were chosen randomly, with the proviso that they were different letters. The last item in the RSVP was always a distractor digit. Following the disappearance of this digit, a $200-\mathrm{ms}$ blank screen was presented, and then participants were prompted to type in the letters that they had seen during the trial. Participants were instructed to enter the letters in any order, to guess if they were sure that a letter had appeared but they did not know its identity, or to press the space bar if they thought that the second target had not been presented. After making their responses, the fixation cross reappeared, and participants could initiate the next trial.

\section{Results}

The main finding was that $\mathrm{T} 2$ accuracy was significantly greater at the longest lag (lag 7) in the aging than in the nonaging condition. However, T2 accuracy was not significantly greater in the nonaging than in the aging condition at the shortest lag (lag 2). This pattern of results suggests that participants were sensitive to the temporal cues provided by the statistical patterns in the aging conditions (i.e., the foreperiod effect), but not to differences in the a priori probabilities of trials at each lag, as had been found in earlier studies by Martens and Johnson (2005) and Visser et al. (2014).

The mean target accuracy was first calculated separately for each lag in each condition. Only performance on the last $80 \%$ of trials was considered, on the grounds that differences in probability could only be established after exposure to a number of trials. That said, the pattern of results reported below was also found when all trials were considered in the analysis. As has been customary in many studies of the $\mathrm{AB}$, secondtarget accuracy was calculated only on trials in which $\mathrm{T} 1 \mathrm{had}$ been identified correctly, in an effort to ensure that the first target had been attended (Raymond et al., 1992). Preliminary examination of the data showed that one participant had an overall $\mathrm{T} 1$ accuracy more than 2.5 standard deviations below the group mean, and thus the data from this participant were omitted from further analyses. This left a sample of 19 participants. 
T1 performance Mean $\mathrm{T} 1$ accuracy scores, shown in Table 1, were submitted to a Condition (aging, nonaging) $\times \operatorname{Lag}(2,3$, $5,7)$ within-subjects analysis of variance (ANOVA). The analysis revealed no significant main effects or interactions ( $p \mathrm{~s}>$ $.76, \eta^{2} \mathrm{~s}<.022$ ), indicating that neither trial distribution nor lag influenced $\mathrm{T} 1$ accuracy.

T2 performance In order to examine the impact of condition on the $\mathrm{AB}$, we first calculated an index of $\mathrm{AB}$ magnitude separately for each lag by subtracting T2 $\mid \mathrm{T} 1$ scores from T1 scores (as is shown in Table 1). These means are illustrated in Fig. 1. We chose to analyze $\mathrm{AB}$ magnitude rather than conditional $\mathrm{T} 2$ accuracy on the grounds that it better isolates the contribution of attention to T2 performance (although the same pattern of results was obtained if conditional T2 accuracy was analyzed). Conditional $\mathrm{T} 2$ accuracy reflects the specific costs associated with attending to T1 as well as more general perceptual and cognitive abilities required to perceive items presented in rapid succession. Because the impact of these general abilities can be estimated by considering $\mathrm{T} 1$ accuracy, it then follows that calculating a difference score between $\mathrm{T} 1$ and $\mathrm{T} 2$ at each lag will isolate the contribution of attentional availability to $\mathrm{T} 2$ performance. We submitted these difference scores to a Condition $\times$ Lag within-subjects ANOVA, which yielded a significant main effect of lag, $F(3$, $54)=15.80, p<.001, \eta^{2}=.47$, indicating robust $\mathrm{ABs}$ in both conditions, a marginally significant main effect of condition, $F(1,18)=3.42, p=.081, \eta^{2}=.16$, and a nonsignificant Lag $\times$ Condition interaction, $F(3,54)=1.49, p>.22, \eta^{2}<.08$.

Although the interaction was nonsignificant, on the basis of past literature, we hypothesized in the introduction that a significant difference in T2 accuracy across conditions should be found at the shortest lag, due to the large change in the a priori probability of $\mathrm{T} 2$ across conditions, and at the longest lag, due to the foreperiod effect. For this reason, and because differences specific to these two lags might have been obscured in the context of the overall ANOVA that included all four lags run in the experiment, we performed additional analyses specifically comparing performance in the nonaging and aging conditions at lags 2 and 7. As is suggested by examination of Fig. 1, AB magnitude was significantly greater in the nonaging than in the aging condition at lag $7, t(18)=2.82, p=.011$.

Table 1 Mean percentage accuracies for T1 and T2 | T1, separated by condition and lag in Experiment 1

\begin{tabular}{llllll}
\hline Condition & Target & Lag 2 & Lag 3 & Lag 5 & Lag 7 \\
\hline \multirow{2}{*}{ Aging } & T1 & $95.4(0.8)$ & $96.3(1.0)$ & $95.3(0.9)$ & $95.6(1.0)$ \\
& T2 | T1 & $78.5(2.7)$ & $82.9(2.9)$ & $92.0(2.1)$ & $94.8(1.6)$ \\
\multirow{2}{*}{ Nonaging } & T1 & $95.0(1.1)$ & $96.0(1.2)$ & $96.0(1.2)$ & $96.3(1.4)$ \\
& T2|T1 & $76.4(3.3)$ & $81.1(2.7)$ & $92.7(1.7)$ & $90.3(2.1)$ \\
\hline
\end{tabular}

Numbers in parentheses represent one standard error of the mean.

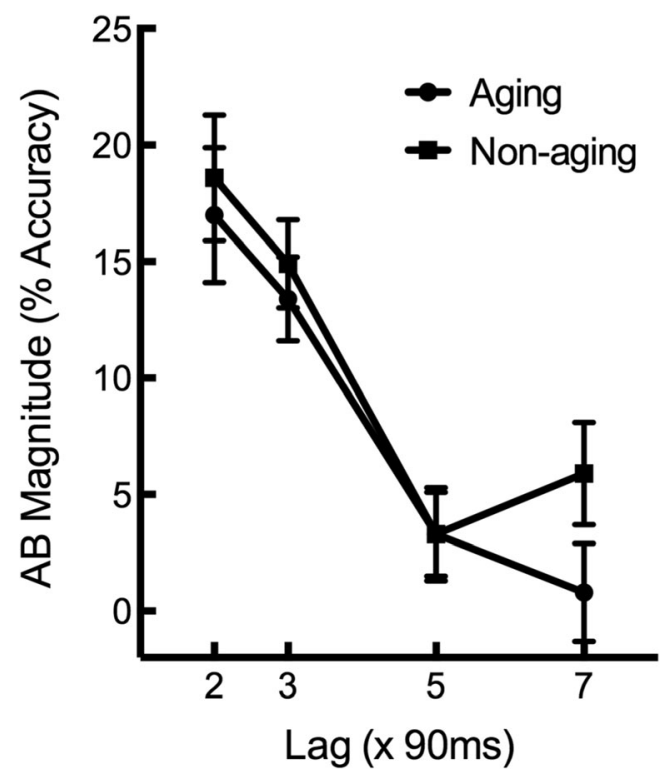

Fig. 1 Magnitudes of the attentional blink deficit (AB, calculated by subtracting T2 | T1 from T1 accuracy separately at each lag) in Experiment 1, plotted as a function of intertarget lag. Solid circle symbols represent $\mathrm{AB}$ magnitudes in the aging condition, and solid square symbols represent $\mathrm{AB}$ magnitudes in the nonaging condition. Error bars represent one standard error of the mean

However, this difference was not significant at lag $2, t(18)=$ $0.84, p>.41$.

The lack of a significant difference in performance between conditions at lag 2 suggests that participants were insensitive to changes in the a priori probability of trials at particular lags. This result parallels earlier findings by Martens and Johnson (2005) and Visser et al. (2014), who both showed that AB magnitude was unaffected by presenting a block of trials at a consistent lag when participants were uninformed of this contingency. On the other hand, the pattern of performance at lag 7 suggests that participants are sensitive to statistical pattern information that arises from using equal numbers of trials at each lag. When this information was eliminated by reducing the number of trials at lag 7 in the nonaging condition, the $A B$ magnitude increased significantly.

That said, it is important to note that the greater $\mathrm{AB}$ magnitude in the nonaging condition at lag 7 could have arisen either due to the elimination of statistical pattern information or because of a reduction in the a priori probability of trials occurring at this lag. Thus, it is not yet clear whether the performance differences at lag 7 are evidence for participants' sensitivity to the temporal information provided by statistical patterns in the aging condition or to reductions in the a priori probability of lag-7 trials in the nonaging condition. To address this question, in Experiment 2, we replicated the paradigm used in Experiment 1, but eliminated catch trials. This change equated the temporal information provided by the passage of time following $\mathrm{T} 1$ at lag 7 (in both conditions, the probability of $\mathrm{T} 2$ appearing at lag 7 , given that it had not 
appeared at an earlier lag, was 1.0), while maintaining differences in the a priori probabilities of lag 7 trials.

The predictions were straightforward. If the results at lag 7 in Experiment 1 were indicative of sensitivity to temporal information in aged distributions, then the difference in $\mathrm{AB}$ magnitudes between the aging and nonaging conditions should be eliminated in Experiment 2. On the other hand, if the differences at lag 7 in Experiment 1 were due to sensitivity to differences in the a priori probability of trials at a given lag, then $\mathrm{AB}$ magnitude should remain greater in the nonaging condition in Experiment 2.

\section{Experiment 2}

\section{Method}

Participants Twenty undergraduate students (13 female, seven male; mean age 18.38 years) were recruited in exchange for partial credit toward course completion. All provided informed consent prior to participating, had normal or corrected-to-normal vision, and were naïve as to the purpose of the experiment. None had participated in Experiment 1.

Apparatus and stimuli The apparatus and stimuli were identical to those of Experiment 1, except that stimuli were presented on a 24-in. BenQ XL2420TE monitor.

Procedure The procedure was similar to that of Experiment 1, except that catch trials were eliminated, yielding a total of 240 trials in each condition. As in Experiment 1, 60 trials were presented at each lag in the aging condition. Given this distribution of trials, the probability that $\mathrm{T} 2$ would be presented at lag 2 was .25, at lag 3 was .33, at lag 5 was .50, and at lag 7 was 1.0 . In the nonaging condition, 124 trials were presented at lag 2,64 trials at lag 3, 32 trials at lag 5, and 20 trials at lag 7. Given this distribution of trials, the probability that T2 would be presented at lag 2 (given that it had not already appeared) was .52, at lag 3 was .51, at lag 5 was .52, and at lag 7 was 1.0. Note that the a priori probability of T2 being presented at lag 7 was .25 in the aging condition and .083 in the nonaging condition. This closely matched the a priori probability of lag-7 trials in the aging and nonaging conditions in Experiment 1, as well as the magnitude of the probability difference between these conditions.

\section{Results}

The main finding was that $\mathrm{AB}$ magnitudes did not differ between conditions at any lag. This pattern of results suggests that participants are sensitive to the statistical patterns inherent in aging distributions and that this sensitivity drove the performance differences seen at lag 7 in Experiment 1.
Additionally, as in Experiment 1, despite similar differences in a priori probabilities between the aging and nonaging conditions at lag 2, we again found no differences in $\mathrm{AB}$ magnitude.

As in Experiment 1, the mean target accuracy was first calculated separately for each lag in each condition, considering only the last $80 \%$ of trials. Preliminary examination of the data showed that one participant had an overall T1 accuracy more than 2.5 standard deviations below the group mean, and thus the data from this participant were omitted from further analyses. This left a sample of 19 participants.

T1 performance Mean T1 accuracy scores, shown in Table 2, were submitted to a Condition $\times$ Lag within-subjects ANOVA. The analysis revealed no significant main effects or interactions ( $p \mathrm{~s}>.23, \eta^{2} \mathrm{~s}<.08$ ), indicating that neither trial distribution nor lag influenced T1 accuracy.

T2 performance AB magnitude scores were submitted to a Condition $\times$ Lag within-subjects ANOVA. As is suggested by examination of these scores in Fig. 2, this analysis yielded only a significant main effect of lag, $F(3,54)=14.67, p<$ $.001, \eta^{2}=.45$, indicating robust $\mathrm{ABs}$ in both conditions. No other main effects or interactions were significant ( $p s>.26$, $\eta^{2} \mathrm{~s}<.07$ ). Follow-up $t$ tests comparing performance between the conditions separately at lags 2 and 7 also were nonsignificant $[\operatorname{lag} 2, t(18)=0.27, p>.78 ; \operatorname{lag} 7, t(18)=1.07, p>.29]$.

Considered together with the findings from Experiment 1, these results provide clear evidence that participants are sensitive to the temporal information given by the passage of time since $\mathrm{T} 1$ in the aging condition. This indicates that participants can derive temporal cues from statistical patterns and can use this information to ameliorate the $\mathrm{AB}$, in much the same way that participants are sensitive to other statistical patterns that communicate information about the likely location and identity of upcoming sensory inputs (Turk-Browne et al., 2009).

The results from Experiment 2 also bolster the evidence obtained in Experiment 1 and in earlier studies suggesting that participants are insensitive to temporal information derived from differences in the a priori probabilities of trials occurring at particular lags. One possible explanation for this insensitivity is that the differences in probability are not sufficiently

Table 2 Mean percentage accuracies for T1 and T2|T1, separated by condition and lag in Experiment 2

\begin{tabular}{llllll}
\hline Condition & Target & Lag 2 & Lag 3 & Lag 5 & Lag 7 \\
\hline Aging & T1 & $96.0(0.7)$ & $97.2(0.6)$ & $97.3(0.5)$ & $96.7(0.6)$ \\
& T2 | T1 & $80.9(3.0)$ & $86.4(2.1)$ & $96.0(1.0)$ & $95.8(0.6)$ \\
\multirow{2}{*}{ Nonaging } & T1 & $96.3(0.6)$ & $96.6(0.7)$ & $97.6(0.8)$ & $97.5(0.7)$ \\
& T2|T1 & $81.7(3.2)$ & $85.4(2.9)$ & $93.6(1.2)$ & $94.8(1.1)$ \\
\hline
\end{tabular}

Numbers in parentheses represent one standard error of the mean. 


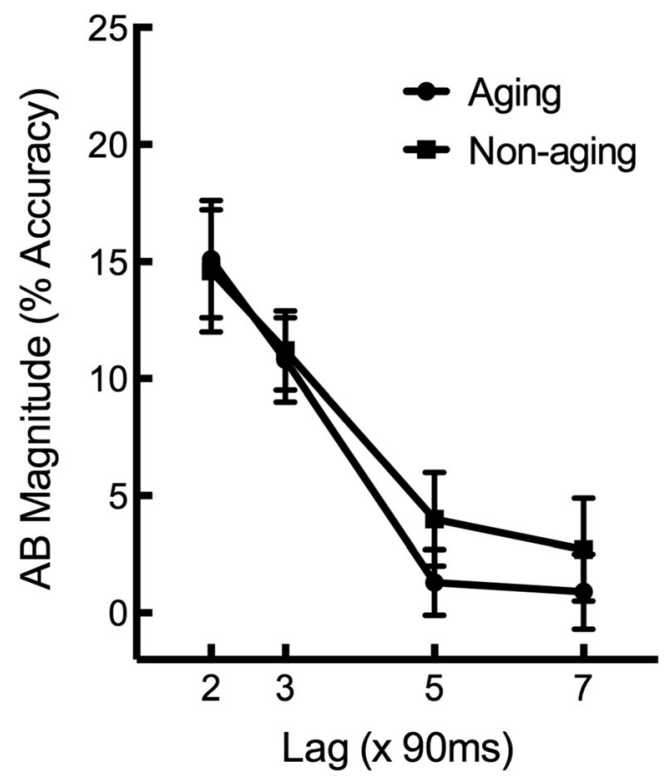

Fig. 2 Magnitudes of the attentional blink deficit (AB, calculated by subtracting T2 | T1 from T1 accuracy separately at each lag) in Experiment 2, plotted as a function of intertarget lag. Solid circle symbols represent $\mathrm{AB}$ magnitudes in the aging condition, and solid square symbols represent $\mathrm{AB}$ magnitudes in the nonaging condition. Error bars represent one standard error of the mean

salient to be recognized. Support for this option has come from Visser et al. (2014), who found that informing participants about the presence of blocks of trials at a consistent lag increased T2 accuracy. Another possibility is that probabilitylinked effects are too small to be detected using a measure of T2 accuracy. In light of this option, a more sensitive alternative measure might be $\mathrm{T} 2$ response times (RTs). The rationale for this suggestion is twofold. First, RTs largely avoid potential problems with scale constraints - in particular, ceiling effects. This is particularly salient here, because T2 accuracy was relatively high across lags. Second, previous studies had suggested that temporal information can significantly benefit response-related processes (e.g., Coull \& Nobre, 1998). Thus, any temporal information accrued from consideration of the a priori probability of trials at a particular lag might be more likely to benefit performance when $\mathrm{T} 2$ requires a speeded judgement.

To test this second possibility, we modified the AB task to require a speeded two-alternative forced choice discrimination for T2 (presented without a trailing mask), allowing us to estimate T2 processing time directly (Visser, 2007). This task is similar to those used by Jolicœur and Dell'Acqua (1998) and Zuvic, Visser, and Di Lollo (2000), who both found an $\mathrm{AB}$ reflected in a decrease in $\mathrm{T} 2 \mathrm{RTs}$ with increasing lag. In order to bolster our statistical power, we also conducted a power analysis (Faul, Erdfelder, Lang, \& Buchner, 2007) using the effect sizes and intercorrelations amongst levels of the repeated measures variables obtained in Experiment 1, in order to estimate the sample size required for approximately
.95 power. This yielded an estimate of 44 participants. Of chief interest was whether using a more sensitive RT measure, combined with a larger sample size, would yield evidence for changes in $\mathrm{AB}$ magnitude reflective of the a priori probability of trials occurring at a given lag.

\section{Experiment 3}

Method

Participants Forty-five undergraduate students (30 female, 15 male; mean age 19.62 years) were recruited in exchange for partial credit toward course completion. All provided informed consent prior to participating, had normal or correctedto-normal vision, and were naïve as to the purpose of the experiment. None had participated in Experiment 1 or 2.

Apparatus and stimuli The apparatus and stimuli were identical to those of Experiment 2, except that some participants viewed stimuli on a 19-in. Acer AC716 monitor.

Procedure The procedure was identical to that of Experiment 1 , with three exceptions. First, T2 was equally likely to be a G or $\mathrm{C}$ on each trial, whereas $\mathrm{T} 1$ was additionally constrained to exclude $\mathrm{G}$ or $\mathrm{C}$. Second, the last distractor was eliminated, so that each trial ended with T2. Third, participants were instructed to respond as quickly and accurately as possible to the appearance of $\mathrm{T} 2$ by pressing an appropriately marked key on the keyboard and then to respond to T1 at their leisure when prompted. On trials on which T2 was omitted, participants were asked to wait for the T1 prompt without responding. This prompt appeared 2,000 ms following the presentation of $\mathrm{T} 2$ if no key was pressed.

\section{Results}

The main finding was a crossover interaction in RTs, such that they were faster at lag 2 in the nonaging condition (over the aging condition), and faster at lag 7 in the aging condition (over the nonaging condition). The faster nonaging RTs at lag 2 indicate sensitivity to the a priori probability of $\mathrm{T} 2 \mathrm{oc}-$ curring at this lag. The faster aging RTs at lag 7 provide converging evidence that the distribution of trials in this condition provides a reliable statistical pattern that can be used to aid T2 processing as the time since $\mathrm{T} 1$ onset increases.

As in Experiments 1 and 2, the mean target accuracy and median T2 RTs were calculated separately for each lag in each condition, using only the final $80 \%$ of trials. Mean T1 and T2 | T1 accuracy scores are shown in Table 3 . The data from four participants whose overall $\mathrm{T} 1$ accuracy scores were more than 2.5 standard deviations below the group mean were omitted from further analysis. The data from one participant whose 
Table 3 Mean percentage accuracies for T1 and T2 | T1, separated by condition and lag in Experiment 3

\begin{tabular}{llllll}
\hline Condition & Target & Lag 2 & Lag 3 & Lag 5 & Lag 7 \\
\hline Aging & T1 & $91.0(1.2)$ & $91.1(1.2)$ & $91.0(1.2)$ & $89.9(1.2)$ \\
& T2 | T1 & $96.5(0.5)$ & $97.6(0.5)$ & $98.1(0.4)$ & $98.1(0.4)$ \\
\multirow{2}{*}{ Nonaging } & T1 & $92.3(0.8)$ & $92.3(0.9)$ & $91.3(1.0)$ & $91.0(1.3)$ \\
& T2 | T1 & $97.1(0.4)$ & $97.2(0.4)$ & $96.8(0.7)$ & $98.1(0.7)$
\end{tabular}

Numbers in parentheses represent one standard error of the mean.

overall $\mathrm{T} 2$ accuracy score in the aging condition was more than four standard deviations below the group mean were also omitted.

T1 performance Mean T1 accuracy scores were submitted to a Condition $\times$ Lag within-subjects ANOVA. The analysis did not reveal any significant main effects or interactions ( $p$ s $>$ $\left..21, \eta^{2} \mathbf{s}<.04\right)$, indicating no effect of trial distribution or lag on $\mathrm{T} 1$ accuracy.

T2 performance (accuracy) As can be seen in Table 3, mean T2 $\mid$ T1 accuracy scores were much higher than in previous experiments, due to the omission of the mask after T2. These means were submitted to Condition $\times$ Lag within-subjects ANOVA that revealed only a main effect of lag, $F(3,117)=$ $3.04, p=.032, \eta^{2}=.07$, consistent with small ABs across both conditions. No other significant main effects or interactions $\left(p \mathrm{~s}>.10, \eta^{2} \mathrm{~s}<.06\right)$ were obtained.

T2 performance (adjusted RTs) Trials on which RTs were less than $200 \mathrm{~ms}$ or greater than 2,000 ms were excluded from the analysis, since they indicated that participants had either anticipated target onset or failed to respond to $\mathrm{T} 2$ before the prompt requesting $\mathrm{T} 1$ identity had been displayed. This resulted in the omission of $1.10 \%$ of trials across all conditions. In order to examine the impact of trial probability on the $\mathrm{AB}$, we calculated adjusted RTs separately at each lag by dividing the median RTs by the proportion of correct responses. The purpose of adjusted RTs is to provide a more sensitive, composite index of target perceptibility that takes into account both response speed and criterion (Chambers, Stokes, Janko, \& Mattingley, 2006; Townsend \& Ashby, 1983). This overcomes the conventional requirement to consider accuracy and RTs separately, and the associated uncertainties about which is the superior indicator of perceptual sensitivity. Notably, the pattern of adjusted RTs reported below was mirrored when considering RTs alone.

We submitted the adjusted RTs to a Condition $\times$ Lag within-subjects ANOVA. The main effect of condition was not significant, $F(1,39)=0.04, p>.83, \eta^{2}<.01$. However, as is evidenced in Fig. 3, we found a main effect of lag, $F(3$, $117)=39.51, p<.001, \eta^{2}=.50$, consistent with an overall

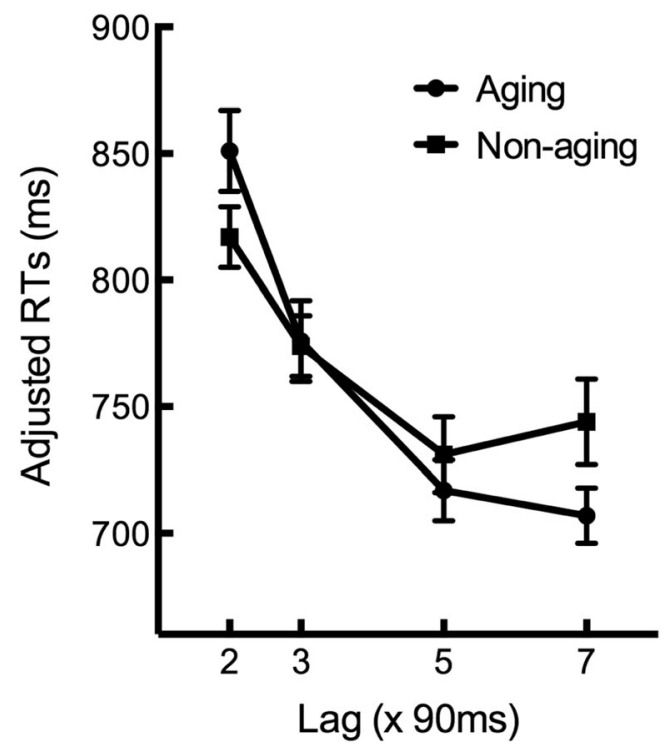

Fig. 3 Mean adjusted response times (RTs) in Experiment 3, plotted as a function of intertarget lag. Solid circle symbols represent performance in the aging condition, and solid square symbols represent performance in the nonaging condition. Error bars represent one within-subjects standard error of the mean, calculated using the Cousineau-Morey method (Cousineau, 2005; Morey, 2008)

drop in adjusted RTs with increasing lag. This is the signature of the AB deficit seen in previous research (Jolicœur \& Dell'Acqua, 1998; Zuvic et al., 2000). Moreover, there was a significant Condition $\times$ Lag interaction, $F(3,117)=6.65, p<$ $.001, \eta^{2}=.15$. As in earlier analyses, we followed up the ANOVA by comparing performance across the aging and nonaging conditions at lags 2 and 7. Examination of Fig. 2 suggests that performance was faster in the nonaging condition than in the aging condition at lag 2 , but that this pattern reversed at lag 7. Consistent with this impression, significant differences in adjusted RTs emerged at lag $2, t(39)=1.73, p=$ .046 (one-tailed), and at lag 7, $t(39)=2.03, p=.049$ (twotailed). This pattern replicates the findings of Experiment 1, showing that participants are sensitive to the temporal cues provided by statistical patterns arising in the aging condition. The results also show evidence that participants are sensitive to the temporal information provided by differences in the numbers of trials presented at each lag across the experiment. However, even when we boosted the likelihood that these cues would be helpful by requiring a speeded response to $\mathrm{T} 2$, the improvements were quite modest.

\section{General discussion}

A number of studies have examined the interaction between temporal cues and attentional limits using the $\mathrm{AB}$ paradigm, in which processing of a second target is impaired by the requirement to attend to an immediately preceding item. This work has shown reliable effects of explicit temporal cues (e.g., 
Hilkenmeier \& Scharlau, 2010; Martens \& Johnson, 2005), as well as salient events within the task that indicate the likely appearance of T2 (e.g., Nieuwenstein et al., 2005). However, results have been mixed when participants have not received salient temporal cues and/or no explicit instruction (Badcock et al., 2013; Martens \& Johnson, 2005; Tang et al., 2014). This study has clarified the apparent lack of consistency on this issue by demonstrating that participants can use the predictive temporal information contained in the statistical structure of a trial block in order to overcome limitations of attention.

Here, we investigated the source of these conflicting results by examining whether $\mathrm{AB}$ task performance is influenced by a foreperiod effect (Niemi \& Näätänen, 1981). We compared a standard $\mathrm{AB}$ task condition (in which all potential intertarget lags occurred with equal frequencies) with one in which trials at shorter lags were more frequent. In the standard design, the factor of Lag occurs with an "aging" distribution, such that T2 is increasingly likely to occur as time passes from the onset of T1. This was compared with a "nonaging" distribution, in which the frequency of T2 trials at each lag was reduced by approximately half as lag increased (Näätänen, 1970). This difference in frequency between conditions also produced variations in the a priori probability that a trial at a particular lag would be presented, similar to earlier experiments in which performance on blocks of trials presented at randomly varying lags was compared to performance on blocks of trials presented at a constant lag (Martens \& Johnson, 2005; Visser et al., 2014). Thus, in our experiment, we were able to assess participants' sensitivity to two different types of temporal information embedded in the trial structure of the $\mathrm{AB}$ task.

On the whole, the present results are in line with previous studies showing that target performance is enhanced by the presence of a consistent interval prior to T1 (Badcock et al., 2013), as well as a broader literature suggesting humans are highly sensitive to statistical patterns and environmental regularities (Turk-Browne et al., 2009; Zhao et al., 2013). The results of Experiments 1 and 3 revealed a strong foreperiod effect at the longest lag in the aging condition, suggesting that participants could make some predictions about T2 onset on the basis of the temporal information afforded by the distribution of lags within a condition. With a more sensitive RT measure of T2 processing in Experiment 3, the results showed that participants could also benefit from temporal predictions based on the a priori probability of different lags across a block of trials. This indicates that the temporal information concerning lag that is contained in a block of trials can be used effectively even when these regularities are not communicated explicitly (Visser et al., 2014).

However, this conclusion does come with caveats. First, the fact that variations in the relative frequencies of an intertarget lag on T2 performance in Experiment 3 were detectable only with a more sensitive RT measure and larger sample size suggests that this type of temporal information leads to relatively subtle effects that may vary with the sensitivity of the performance measure and the nature of the underlying processes that it taps. Second, it is clear that participants are differentially sensitive to sources of temporal information contained in the statistical patterns present in the $\mathrm{AB}$ task. Whereas participants reliably showed foreperiod effects, probability variations were much more modest. Considering these results and past demonstrations of the benefits of explicitly informing participants about the existence of temporal information, it seems reasonable to conclude that explicit information likely boosts temporal cuing benefits but are not necessary for such effects to be found. Additionally, it is clear that future work will be needed to examine the interaction between participants' knowledge about the presence of temporal information in a task and the type of temporal information in order to gain a more complete picture of how and when such information is used.

An obvious question is why temporal cue information arising from aging distributions was considerably stronger than those arising from variations in a priori probability. Some explanation for this difference may lie in the fact that foreperiod effects are mediated by multiple mechanisms, only one of which was likely to have been engaged by variations in a priori probability. The foreperiod effect has been suggested to arise from (a) increased alertness induced by the warning signal, in combination with increasing certainty about target appearance as time passes (Bertelson, 1967; Coull \& Nobre, 1998); (b) deployment of attentional resources at the expected target interval (Correa, Lupiáñez, Milliken, \& Tudela, 2004; Coull \& Nobre, 1998); and (c) trace conditioning, wherein the warning signal initiates a preparatory RT locked to target appearance (Los \& Heslenfeld, 2005; Los \& Van den Heuvel, 2001). Notably, the operation of the first and last of these mechanisms requires a high degree of temporal certainty. However, this is unlikely to result from changes in a priori probability. For example, lag-2 trials in the nonaging condition still only occurred on approximately half of the trials, and this was little different from the corresponding lag in the aging condition (see below). As a result, temporal cues arising from variations in a priori probability are less likely than those arising from aging distributions to induce increased alertness or trace conditioning. In turn, cuing benefits are likely to be smaller in the former than in the latter case.

Another issue addressed by the present results concerns the degree to which the a priori probabilities of an event must differ in order for this information to serve as a temporal cue. In Martens and Johnson (2005), two lags were used and the cue was either present or absent. Thus, the a priori probability of a trial presented at the shortest lag in their experiment was either 1.0, with the cue present, or .50, when the cue was absent - a difference of .50. The present results augment this initial finding by showing that temporal information can also be gleaned from smaller variations in target predictability. For 
example, in Experiment 3, the a priori probability of a lag-2 trial was approximately .23 in the aging condition, and .50 in the nonaging condition - a difference about half that in Martens and Johnson. Future investigations into this issue would benefit from looking beyond group means, as we have done here, in order to investigate individual differences in sensitivity to the probability information available in the environment. This would provide a valuable new perspective on our ability to use the predictive information available from task structure to guide perception.

To the extent that participants can predict the onset of $\mathrm{T} 2$, how does this benefit performance? A variety of suggestions have been made in this regard. Martens and Johnson (2005) argued that when $\mathrm{T} 2$ was presented at a cued interval, it gained additional weighting in a postperceptual competition with adjacent items (particularly T1) for high-level resources. Alternatively, Visser et al. (2014) proposed that cueing T2 onset allows more efficient suppression of distractors by increasing the efficiency of a perceptual filtering process (Ghorashi, Zuvic, Visser, \& Di Lollo, 2003; Visser, Bischof, \& Di Lollo, 2004) that screens out distracting inputs prior to engagement with postperceptual resources. Although the present study did not address this issue directly, we believe the present results are consistent with distractor suppression, because the finding of a foreperiod effect at long lags in Experiments 1 and 3 likely occurred after T1 processing had already been completed. This leaves more effective suppression of the stream of distractors as a viable mechanism, in addition to the possibility that temporal cuing may benefit $\mathrm{T} 2$ in the competition for attentional resources with T1 at short lags.

As we noted in the introduction, all theories of the $A B$ attribute the deficit to the requirement to process $\mathrm{T} 1$. However, there are important differences between these accounts in terms of the putative mechanisms underlying this relationship. According to bottleneck accounts, the AB arises because the processing resources allocated to $\mathrm{T} 1$ are unavailable for processing T2 when it is presented at shorter lags (e.g., Chun \& Potter, 1995; Jolicœur \& Dell'Acqua, 1998). On the other hand, according to distractor-based accounts, presentation of nontarget items following T1 initiates mechanisms designed to suppress perceptual processing in order to prevent interference with T1 (e.g., Olivers \& Meeter, 2008; Taatgen et al., 2009; Wyble et al., 2009). This interferes with $\mathrm{T} 2$ processing at short intertarget intervals.

The present results indicate that limits on $\mathrm{T} 2$ processing can be overcome by virtue of the temporal information provided by statistical patterns in the $A B$ task, even when these patterns are not explicitly communicated to participants. In past studies, it has been suggested that temporal cues reduce postperceptual competition between targets and distractors by boosting the strength of the target representation (Martens \& Johnson, 2005) and/or by allowing perceptual resources to be focused on the expected time of T2 arrival, thereby avoiding selection of distractors interposed between T1 and T2. From these perspectives, the present results would seem most consistent with distractor-based accounts of the AB in suggesting that temporal cues reduce or eliminate the deleterious effects of post-T1 distraction.

A final important point to be made regards the implications of the foreperiod effects found here on conventional estimates of the duration of the AB. More than 20 years of studies have indicated that the the impact of $\mathrm{T} 1$ identification on the processing of $\mathrm{T} 2$ in neurotypical populations extends for approximately 500-700 ms. On this basis, the negative impact of stimulus processing on perception is conventionally assumed to cease after about $700 \mathrm{~ms}$. However, almost without exception, the past studies upon which this conclusion has been based used intertarget lags that occurred with equal frequencies across experimental trials (i.e., an aging distribution with respect to lag). The evidence shown here clearly indicates that this practice leads to a foreperiod effect that has boosted accuracy at the longest lag in these experiments. In turn, this implies that $\mathrm{T} 1$ processing may still have been ongoing at the longest lag in past experiments (usually lag 7), but the deleterious effects of this processing may have been masked by participants' ability to accurately predict the onset time for T2 at longer intertarget intervals. The upshot is that current estimates of the duration of "the blink" are likely to underestimate the true duration that perception is impacted by the processing of prior stimuli.

\section{References}

Badcock, N. A., Badcock, D. R., Fletcher, J., \& Hogben, J. (2013). The role of preparation time in the attentional blink. Vision Research, 76, 68-76. doi:10.1016/j.visres.2012.10.010

Bausenhart, K. M., Rolke, B., \& Ulrich, R. (2008). Temporal preparation improves temporal resolution: Evidence from constant foreperiods. Perception \& Psychophysics, 70(8), 1504-1514.

Bertelson, P. (1967). The time course of preparation. Quarterly Journal of Experimental Psychology, 19, 272-279.

Biederman, I., Mezzanotte, R. J., \& Rabinowitz, J. C. (1982). Scene perception: Detecting and judging objects undergoing relational violations. Cognitive Psychology, 14, 143-177. doi:10.1016/00100285(82)90007-X

Broadbent, D. E., \& Broadbent, M. H. (1987). From detection to identification: Response to multiple targets in rapid serial visual presentation. Perception \& Psychophysics, 42, 105-113. doi:10.3758/ BF03210498

Burle, B., Tandonnet, C., \& Hasbroucq, T. (2010). Excitatory and inhibitory motor mechanisms of temporal preparation. In A. C. Nobre \& J. Coull (Eds.), Attention and time (pp. 244-255). Oxford, UK: Oxford University Press.

Chambers, C. D., Stokes, M. G., Janko, N. E., \& Mattingley, J. B. (2006). Enhancement of visual selection during transient disruption of parietal cortex. Brain Research, 1097, 149-155. doi:10.1016/j.brainres. 2006.04.084 
Choi, H., Chang, L. H., Shibata, K., Sasaki, Y., \& Watanabe, T. (2012). Resetting capacity limitations revealed by long-lasting elimination of attentional blink through training. Proceedings of the National Academy of Sciences, 109, 12242-12247. doi:10.1073/pnas. 1203972109

Choi, H., \& Watanabe, T. (2014). Can attenuation of attentional blink also evoke removal of repetition blindness? Vision Research, 99, 141147. doi:10.1016/j.visres.2014.02.011

Chun, M. M., \& Jiang, Y. (1998). Contextual cueing: Implicit learning and memory of visual context guides spatial attention. Cognitive Psychology, 36, 28-71. doi:10.1006/cogp.1998.0681

Chun, M. M., \& Potter, M. C. (1995). A two-stage model for multiple target detection in rapid serial visual presentation. Journal of Experimental Psychology: Human Perception and Performance, 21, 109-127. doi:10.1037/0096-1523.21.1.109

Correa, Á., Lupiáñez, J., Milliken, B., \& Tudela, P. (2004). Endogenous temporal orienting of attention in detection and discrimination tasks. Perception \& Psychophysics, 66, 264-278. doi:10.3758/ BF03194878

Correa, Á., Lupiáñez, J., \& Tudela, P. (2005). Attentional preparation based on temporal expectancy modulates processing at the perceptual level. Psychonomic Bulletin \& Review, 12, 328-334. doi:10. 3758/BF03196380

Coull, J. T., \& Nobre, A. C. (1998). Where and when to pay attention: The neural systems for directing attention to spatial locations and to time intervals as revealed by both PET and fMRI. Journal of Neuroscience, 18, 7426-7435.

Cousineau, D. (2005). Confidence intervals in within-subjects designs: A simpler solution to Loftus and Masson's method. Tutorials in Quantitative Methods for Psychology, 1, 42-45.

Di Lollo, V., Kawahara, J., Ghorashi, S. M. S., \& Enns, J. T. (2005). The attentional blink: Resource depletion or temporary loss of control? Psychological Research, 69, 191-200. doi:10.1007/s00426-0040173-x

Dux, P. E., \& Marois, R. (2009). The attentional blink: A review of data and theory. Attention, Perception, \& Psychophysics, 71, 1683-1700. doi:10.3758/APP.71.8.1683

Engström, J., Aust, M. L., \& Viström, M. (2010). Effects of working memory load and repeated scenario exposure on emergency braking performance. Human Factors, 52, 551559 .

Faul, F., Erdfelder, E., Lang, A.-G., \& Buchner, A. (2007). G*Power 3: A flexible statistical power analysis program for the social, behavioral, and biomedical sciences. Behavior Research Methods, 39, 175-191. doi:10.3758/BF03193146

Gabay, S., \& Henik, A. (2008). The effects of expectancy on inhibition of return. Cognition, 106, 1478-1486. doi:10.1016/j.cognition.2007. 05.007

Gabay, S., \& Henik, A. (2010). Temporal expectancy modulates inhibition of return in a discrimination task. Psychonomic Bulletin \& Review, 17, 47-51. doi:10.3758/PBR.17.1.47

Ghorashi, S. M., Zuvic, S. M., Visser, T. A., \& Di Lollo, V. (2003). Focal distraction: Spatial shifts of attentional focus are not required for contingent capture. Journal of Experimental Psychology: Human Perception and Performance, 29, 78-91. doi:10.1037/0096-1523. 29.1.78

Hackley, S. A. (2009). The speeding of voluntary reaction by a warning signal. Psychophysiology, 46, 225-233. doi:10.1111/j.1469-8986. 2008.00716.x

Henderson, J. M. (1991). Stimulus discrimination following covert attentional orienting to an exogenous cue. Journal of Experimental Psychology: Human Perception and Performance, 17, 91-106. doi:10.1037/0096-1523.17.1.91

Hilkenmeier, F., \& Scharlau, I. (2010). Rapid allocation of temporal attention in the attentional blink paradigm. European Journal of Cognitive Psychology, 22, 1222-1234.
Ho, C., Tan, H. Z., \& Spence, C. (2005). Using spatial vibrotactile cues to direct visual attention in driving scenes. Transportation Research Part F, 8, 397-412.

Jolicœur, P., \& Dell'Acqua, R. (1998). The demonstration of short-term consolidation. Cognitive Psychology, 36, 138-202. doi:10.1006/ cogp. 1998.0684

Los, S. A., \& Heslenfeld, D. J. (2005). Intentional and unintentional contributions to nonspecific preparation: Electrophysiological evidence. Journal of Experimental Psychology: General, 134, 52-72. doi:10.1037/0096-3445.134.1.52

Los, S. A., \& Schut, M. L. J. (2008). The effective time course of preparation. Cognitive Psychology, 57, 20-55. doi:10.1016/j.cogpsych. 2007.11.001

Los, S. A., \& Van den Heuvel, C. E. (2001). Intentional and unintentional contributions to nonspecific preparation during reaction time foreperiods. Journal of Experimental Psychology: Human Perception and Performance, 27, 370-386. doi:10.1037/00961523.27.2.370

Martens, S., \& Johnson, A. (2005). Timing attention: Cuing target onset interval attenuates the attentional blink. Memory \& Cognition, 33, 234-240. doi:10.3758/BF03195312

Martens, S., \& Wyble, B. (2010). The attentional blink: Past, present, and future of a blind spot in perceptual awareness. Neuroscience \& Biobehavioral Reviews, 34, 947-957. doi:10.1016/j.neubiorev. 2009.12.005

Morey, R. D. (2008). Confidence intervals from normalized data: A correction to Cousineau (2005). Tutorials in Quantitative Methods for Psychology, 4, 61-64.

Näätänen, R. (1970). The diminishing time-uncertainty with the lapse of time after the warning signal in reaction-time experiments with varying fore-periods. Acta Psychologica, 34, 399-418. doi:10.1016/ 0001-6918(70)90035-1

Nakayama, K., \& Mackeben, M. (1989). Sustained and transient components of focal visual attention. Vision Research, 29, 1631-1647. doi: 10.1016/0042-6989(89)90144-2

Niemi, P., \& Näätänen, R. (1981). Foreperiod and simple reaction time. Psychological Bulletin, 89, 133-162. doi:10.1037/0033-2909.89.1. 133

Nieuwenstein, M. R., Chun, M. M., van der Lubbe, R. H. J., \& Hooge, I. T. C. (2005). Delayed attentional engagement in the attentional blink. Journal of Experimental Psychology: Human Perception and Performance, 31, 1463-1475. doi:10.1037/0096-1523.31.6. 1463

Olivers, C. N. L., \& Meeter, M. (2008). A boost and bounce theory of temporal attention. Psychological Review, 115, 836-863. doi:10. 1037/a0013395

Posner, M. I. (1980). Orienting of attention. Quarterly Journal of Experimental Psychology, 32, 3-25. doi:10.1080/ 00335558008248231

Posner, M. I., Snyder, C. R., \& Davidson, B. J. (1980). Attention and the detection of signals. Journal of Experimental Psychology: General, 109, 160-174. doi:10.1037/0096-3445.109.2.160

Potter, M. C. (1976). Short-term conceptual memory for pictures. Journal of Experimental Psychology: Human Learning and Memory, 2, 509-522. doi:10.1037/0278-7393.2.5.509

Raymond, J. E., Shapiro, K. L., \& Arnell, K. M. (1992). Temporary suppression of visual processing in an RSVP task: An attentional blink? Journal of Experimental Psychology: Human Perception and Performance, 18, 849-860. doi:10.1037/0096-1523.18.3.849

Rolke, B., \& Hofmann, P. (2007). Temporal uncertainty degrades perceptual processing. Psychonomic Bulletin \& Review, 14, 522-526. doi: 10.3758/BF03194101

Sanders, A. F. (1998). Elements of human performance: Reaction processes and attention in human skill. Mahwah, NJ: Erlbaum. 
Shen, D., \& Alain, C. (2011). Temporal attention facilitates short-term consolidation during a rapid serial auditory presentation task. Experimental Brain Research, 215(3-4), 285-292.

Taatgen, N. A., Juvina, I., Schipper, M., Borst, J. P., \& Martens, S. (2009). Too much control can hurt: A threaded cognition model of the attentional blink. Cognitive Psychology, 59, 1-29.

Tang, M. F., Badcock, D. R., \& Visser, T. A. W. (2014). Training and the attentional blink: Limits overcome or expectations raised? Psychonomic Bulletin \& Review, 21, 406-411. doi:10.3758/ s13423-013-0491-3

Townsend, J. T., \& Ashby, F. G. (1983). The stochastic modeling of elementary psychological processes. Cambridge, UK: Cambridge University Press.

Turk-Browne, N. B., Scholl, B. J., Chun, M. M., \& Johnson, M. K. (2009). Neural evidence of statistical learning: Efficient detection of visual regularities without awareness. Journal of Cognitive Neuroscience, 21, 1934-1945. doi:10.1162/jocn.2009.21131

Visser, T. A. W. (2007). Masking T1 difficulty: Processing time and the attentional blink. Journal of Experimental Psychology: Human Perception and Performance, 33, 285-297. doi:10.1037/00961523.33.2.285

Visser, T. A. W., Bischof, W. F., \& Di Lollo, V. (2004). Rapid serial visual distraction: Task-irrelevant items can produce an attentional blink. Perception \& Psychophysics, 66, 1418-1432. doi:10.3758/ BF03195008
Visser, T. A. W., Tang, M. F., Badcock, D. R., \& Enns, J. T. (2014). Temporal cues and the attentional blink: A further examination of the role of expectancy in sequential object perception. Attention, Perception, \& Psychophysics, 76, 2212-2220. doi:10.3758/ s13414-014-0710-7

Visser, T. A. W., Zuvic, S. M., Bischof, W. F., \& Di Lollo, V. (1999). The attentional blink with targets in different spatial locations. Psychonomic Bulletin \& Review, 6, 432-436. doi:10.3758/ BF03210831

Ward, R., Duncan, J., \& Shapiro, K. (1997). Effects of similarity, difficulty, and nontarget presentation on the time course of visual attention. Perception \& Psychophysics, 59, 593-600.

Wyble, B., Bowman, H., \& Nieuwenstein, M. (2009). The attentional blink provides episodic distinctiveness: Sparing at a cost. Journal of Experimental Psychology: Human Perception and Performance, 35, 787-807. doi:10.1037/a0013902

Yeshurun, Y., \& Carrasco, M. (1999). Spatial attention improves performance in spatial resolution tasks. Vision Research, 39, 293-306.

Zhao, J., Al-Aidroos, N., \& Turk-Browne, N. B. (2013). Attention is spontaneously biased toward regularities. Psychological Science, 24, 667-677. doi:10.1177/0956797612460407

Zuvic, S. M., Visser, T. A. W., \& Di Lollo, V. (2000). Direct estimates of processing delays in the attentional blink. Psychological Research, 63, 192-198. 